\title{
Treatment outcomes of HIV/AIDs patients in Federal Medical Centre, Umuahia, Abia State, Nigeria
}

\author{
${ }^{1}$ Dr Nwokeukwu H. I., ${ }^{2}$ Dr Chuku A. ${ }^{1}$ Dr Emma- Ukaegbu U, ${ }^{1}$ Dr Nwogu K.C., \\ ${ }^{I}$ Department of Community Medicine, Federal Medical Centre Umuahia, Abia State, Nigeria \\ ${ }^{2}$ Department of Ophthalmology, federal medical centre Umuahia, Abia State, Nigeria
}

\begin{abstract}
Introduction: Antiretroviral therapy (ART) has been remarkably effective in reducing Human Immunodeficiency Virus (HIV)-associated morbidity and mortality. With the inception of ART the long-term survival in HIV-positive populations appears to be approaching that of the general population.

Aims and objectives: To determine the various outcomes of all patients enrolled into ART between 2008 and 2011 in a tertiary ART center in South Eastern Nigeria.

Methods: This cross-sectional study involved analyzing the records of all newly enrolled HIV positive patients receiving treatment for the 4 year period stated above to determine the various outcomes of the patients that commenced ART in the facility.

Key findings: Total enrolled 3855 and total started on drugs 2480 Known death 135(3.5\%), transfer out 436 (11.3\%), Lost to follow up 163 (4.2\%). Restarted 127 (3.3\%) and treatment stopped 59(1.5\%) while 68\% of the patients were retained on treatment.
\end{abstract}

Key words: Treatment outcome and HIV/AIDs

\section{Introduction}

HIV infection can now be characterized as a manageable chronic condition. Life expectancy has increased for newly diagnosed HIV patients since the inception of combination antiretroviral treatment. Longterm survival in HIV-positive populations with access to effective treatment appears to be approaching that of the general population. ${ }^{1}$ In Nigeria, the 2010 HIV Sero prevalence study recorded an overall prevalence of $4.1 \%$ .$^{2}$ Annual AIDS deaths in Nigeria also increased from 192,000 in 2008 to 217,148 in 2012 with a corresponding rise in new HIV infections from 336,379 to 388,864 in the same time period. ${ }^{2}$ In Sub saharan Africa, Between 2004 and the end of 2011, around 8 million people had initiated ART, leading to dramatic reductions in HIVassociated morbidity and mortality $\mathrm{y}^{3,4}$

Different outcomes exist for patients on ART. Patients on ART might be alive and on treatment in a facility, transferred out, lost to follow-up (LTF), dead or still in care but stopped treatment. ${ }^{5-9}$. Patients categorized as 'alive and on treatment' were enrollees who were still receiving treatment. The second group (transfers) included those who had moved to another facility with the knowledge of the health provider. These two groups were defined as 'retained'. 'Loss To Follow up' comprised patients who missed three scheduled monthly clinic visits or medication pick-ups. ${ }^{5,7,9}$ Those designated as LTF may be alive, dead or truly LTF if all efforts at reaching them using available contact information have failed. For LTF patients who are alive, some might be receiving drugs in the same facility and may even re-enroll as new patients; others for some reason discontinue treatment. Dead patients may have died soon after their last visit while some may have lived longer. ${ }^{10}$ Attrition from ART programs is defined to include patients who died or were lost to follow-up whereas retention is defined to be the opposite of attrition (i.e. 1 - attrition) with transferred out patients were excluded from both the numerator and denominator. ${ }^{11}$ Most mortality occurs early, suggesting that earlier diagnosis and treatment may improve outcomes. Consequences of poor adherence and loss to follow up include not only diminished outcome for the patient, but also the public health threat of multidrug-resistant HIV, and widespread transmission of drug-resistant virus. A major implication of LTF and the need for defaulter tracing is the increased risk of development of resistance to drugs. ${ }^{12}$ A systematic review of ART programs in 13 sub Saharan African countries showed retention rates ranging from 46 to $85 \% .{ }^{13}$ In many sub Saharan Africa studies, a large proportion of the patients present very late for treatment with very poor baseline parameters and thus the higher mortality rates recorded with the patients dying within the first few months of commencing therapy. ${ }^{14,15}$ Treatment outcomes and retention have also been found to worsen in adolescents and young adults than in older adults. ${ }^{16,17}$

\section{Materials and Methods}

Study Area: This study was carried out in Federal Medical Center Umuahia Abia state located in South Eastern Nigeria. FMC Umuahia is the only tertiary health facility in the state capital that renders ART services. The study was for the period between January 2008 and Dec 2011. Abia has an estimated population of 
2,845,380 according to the 2006 census. The major occupations are public service, farming and petty trading. During the study period, all HIV-positive patients assessed as being in WHO Clinical stage 3 or 4, or with a CD4 count $<250$ cells/mm3 (irrespective of WHO staging) were considered eligible for ART. These Eligible patients were enrolled after undergoing pre-treatment adherence counselling, and were subsequently placed on ART. CD4 counts and other necessary investigations were done before commencement of ART and as at when due. All enrollees were also required to make monthly or bimonthly visits to the facility for regular health assessment and drug refill.

\section{Data Collection and analysis}

Analysis was performed on data collected as part of routine programme monitoring and evaluation between 2008 and 2011.Data was under the following headings:-Newly Enrolled, newly initiated and Transferred in . The data was analyzed with Excel and Epi-info version 7 software. Mortality was defined as any death while taking treatment. Loss to follow-up was defined as non-attendance for a period of three months after the last ART prescription had been completed.. Both mortality and loss to follow up were confirmed through family reporting and tracing performed by programme staff.

\section{Results}

In this study, out of a total of 3855 enrolled within the study period a total of 2480 patients were commenced on drugs. The age and sex distribution of study population is as follows, $63 \%$ female adults vs $37 \%$ adult males. Amidst the females $3 \%$ were paediatric while $3 \%$ of the males were within the paediatric age group. Known death 135(3.5\%), transfer out 436 (11.3\%), Lost to follow up $163(4.2 \%)$. Restarted $127(3.3 \%)$ and treatment stopped $59(1.5 \%)$ while $68 \%$ of the patients were retained on treatment.

FIGURE 1: ANNUAL MORTALITY RATES

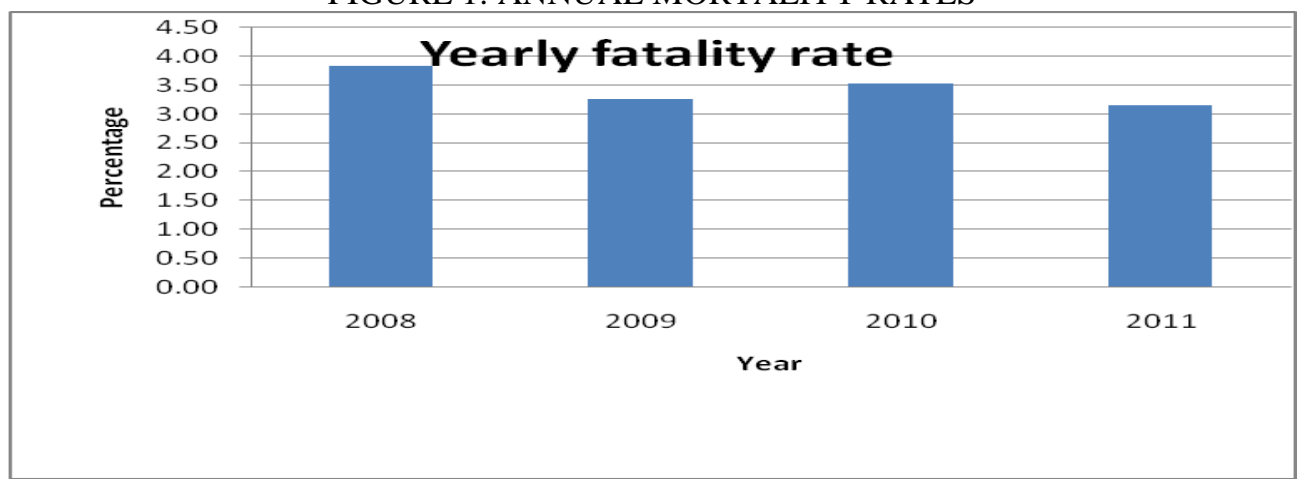

The yearly mortality rate were still within the range of 3.00 and 3.8 highest being in 2008 and lowest in 2011 .

FIGURE 2: The percentage transferred out

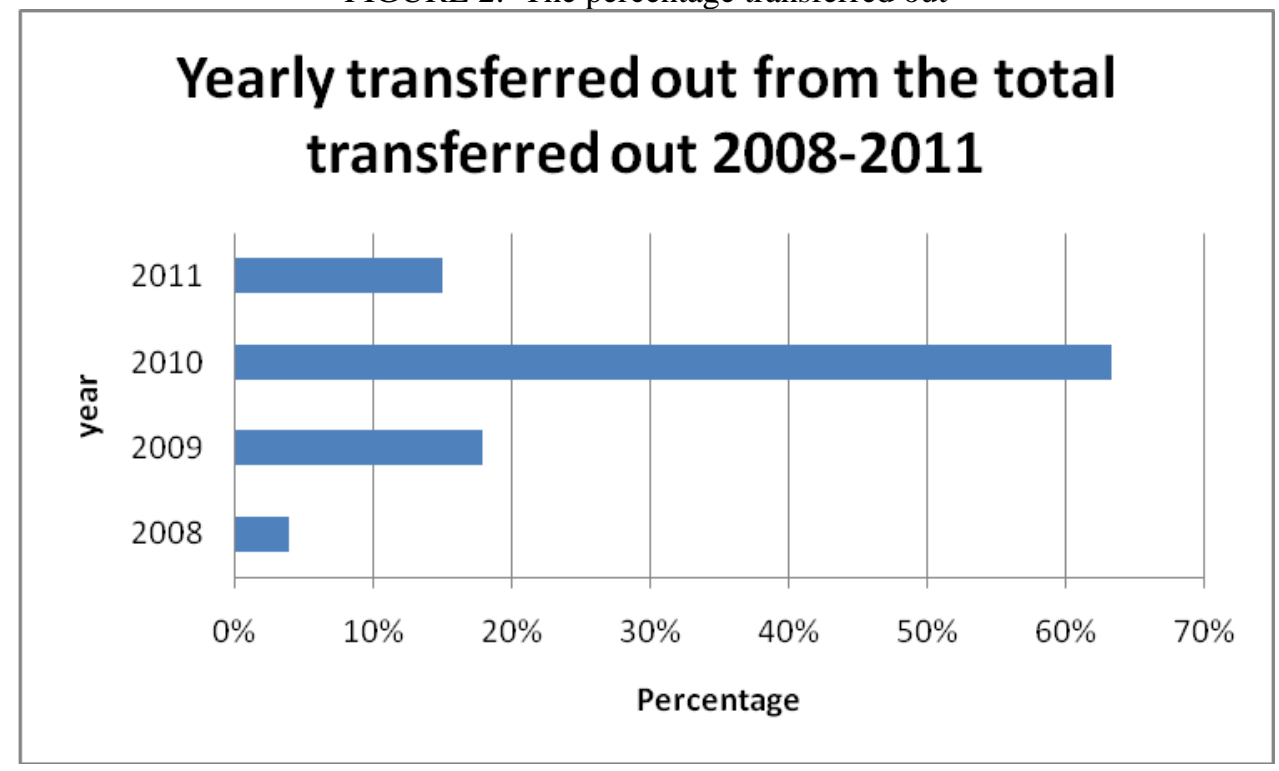

The greatest number of transferred out patients was in year 2010 and lowest in year 2008 . 
FIGURE 3: LOSS TO FOLLOW UP

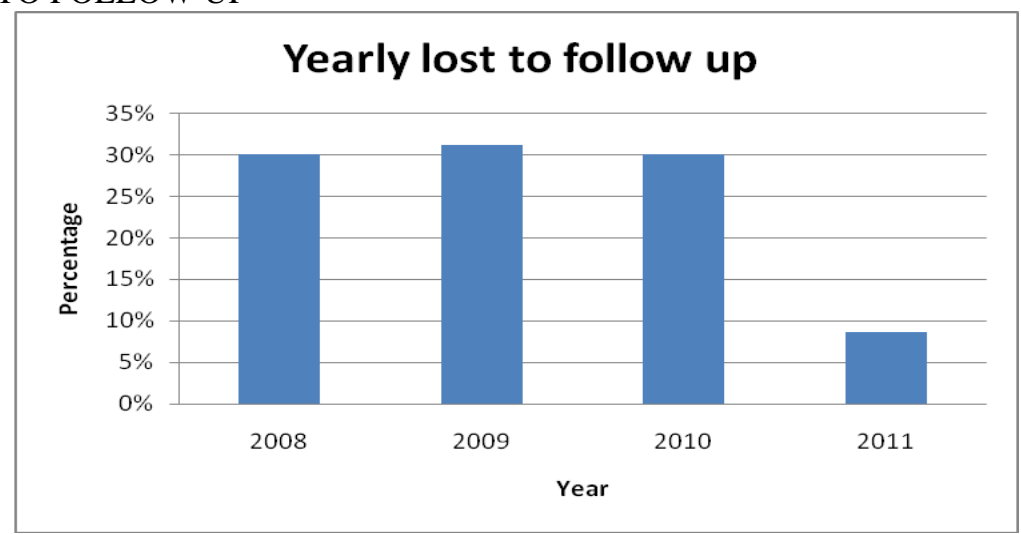

The annual loss to follow up achieved a dramatic improvement between 2010 and 2011 from $30 \%$ to less than $10 \%$.

FIGURE 4: The percentage that restarted treatment

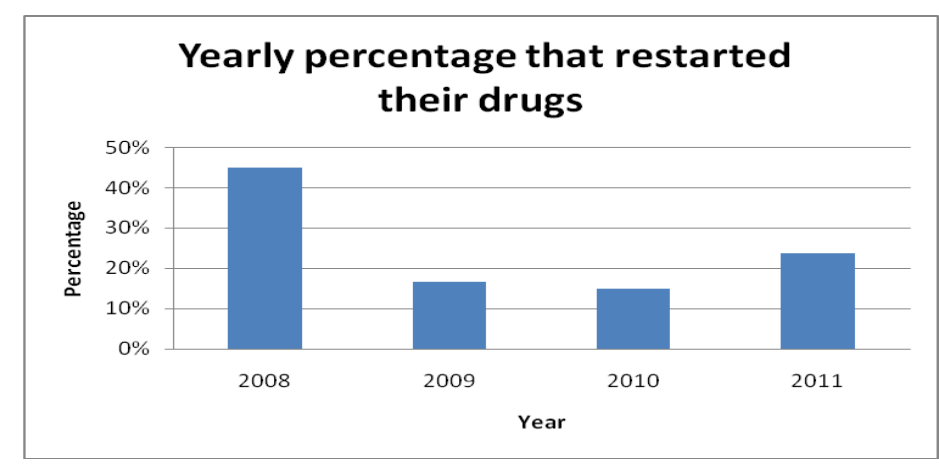

The patients that restarted their drugs were more in year 2010, followed by 2011 and 2009 and 2010 .

FIGURE 5: Treatment stopped

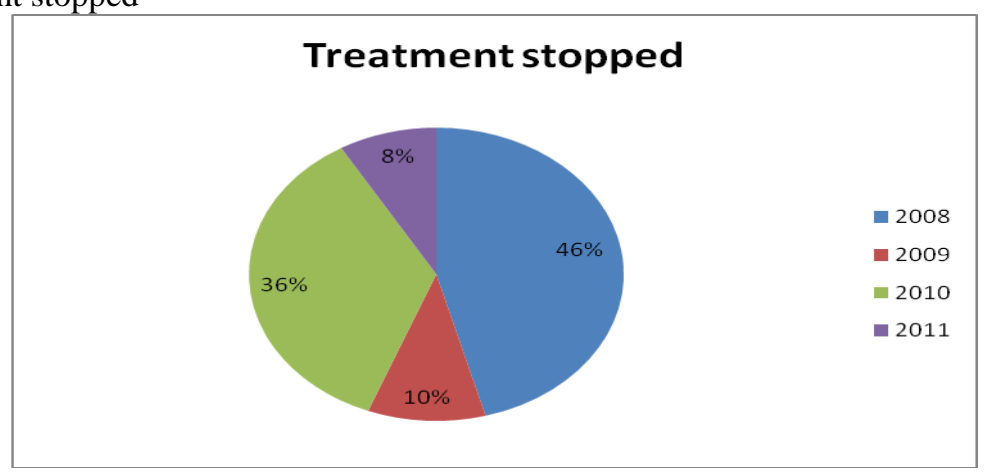

Most of the patients stopped their drugs in 2008(46\%), followed by year 2010(36\%), then 2009(10\%) and $2011(8 \%)$. This is statistically significant with $\mathrm{p}$ value less than .05

\section{Discussion}

Following the commencement of ART, health care workers usually counsel the patients on necessity for adherence, examine patients for clinical progress, provide and monitor combination antiretroviral therapy (ART), and counsel them on achieving better treatment outcomes. However despite these efforts there is need for regular assessment to estimate the different outcomes for those that commenced treatment whether they were lost to follow-up, died, transferred out or stopped treatment. Poor adherence has dire consequences on the general population in terms of spreading drug resistant strains of the virus.

Majority of the patients in this study were females as was found in some other Nigerian studies and Sub Saharan Africa based studies. ${ }^{18,19}$ This could be due to the fact that HCT done in pregnancy provides the women an opportunity to be screened more than their male counterparts and also that females generally have a higher health seeking behaviour than males. It could also because the males are secretly assessing orthodox and non orthodox treatment for their HIV related ill health. 
The mortality rate in this study was lower than most studies conducted, $5.2 \%$ in an SI Bello study, study conducted in South Western Nigeria with a mortality rate of $14.7 \% .^{18,19,20}$ and another study in South East Nigeria had a mortality rate of $6.3 \% .^{10}$

Although the annual mortality rate did not vary much but the lowest was in year 2011 as seen in fig 1 this showed a lot of improvement in the management of the patients, it may not be unrelated to early enrollment and initiation of the treatment with higher CD4 count of 350 cells $/ \mathrm{mm}^{3}$

In this study, lost to follow up was low compared to other studies done. A South African study had $9.8 \%$ of patients being lost to follow-up with $32.8 \%$ mortality rate. ${ }^{21}$ A Nigerian study revealed that $32.8 \%$ of patients receiving ART in public health institutions were lost to follow up while $11 \%$ of private health institution ART receivers were lost to follow up. ${ }^{10} 13.7 \%$ of patients in a Zambian and $6.9 \%$ from Switzerland study were lost to follow up and out of these $9.5 \%$ and $1.6 \%$ of these patients died respectively. ${ }^{22}$ Another Zambian study revealed $7 \%$ mortality and $21 \%$ loss to follow up. It is worthy to note that some patients who are described as been lost to follow up are still receiving treatment in a different facility or have even re started treatment in the same facility due to movements that are not properly notified and documented. In this study, figure 4 above buttresses this fact. Also worthy of note is that some patients who were lost to follow would have entirely stopped receiving medications and as such would be vendors of drug resistant strains of the virus. Lost to follow up reduced drastically in 2011 as shown in fig 3 this could be due to the improvement in health education, meeting of people living with HIV and better tracking of patients.

The transferred out patients were seen most in 2010 fig 2 and this could be due to unavailability of the drugs and the work overload for the limited number of human resources.

The patients that stopped their drugs were lowest in 2011 fig 5. This could be due to reduction in side effects with better combination of anti- retroviral drugs and availability of the drugs.

In this study $68 \%$ of the patients were retained on treatment and similar findings were recorded in a South Eastern Nigerian study with $65.5 \%$ retention rates in a tertiary health facility ${ }^{10}$ and a $61 \%$ retention rate in a Zambian study . ${ }^{23}$

\section{Conclusion and Recommendation}

Findings from studies have shown that proper ART adherence leads to similar life expectancies between PLHIV and normal population. The mortality rate from this study is as low as $3.5 \%$ and loss to follow up as $4.2 \%$. It is however not known what was the final outcome of those that were described as lost to follow up, it is there recommended that a study should be conducted to track the outcome of this population which might further worsen the true mortality picture or who might be busy disseminating drug resistant strains of the virus.

\section{Acknowledgement:}

The workers in the ART centre of Federal Medical Centre, Umuahia are hereby acknowledged for generation and collection of data.

Competing Interest: The authors declare no competing interest.

Approval by the Authors: The authors approved the publication of the manuscript.

\section{References}

[1]. Mahy M, Stover J, Stanecki K, Stoneburner R, Tassie JM . Estimating the impact of antiretroviral therapy: regional and global estimates of life-years gained among adults. Sex Transm Infect 2010, 86 Suppl 2: ii67-71

[2]. Federal Ministry of Health (FMOH). National HIV Sero-prevalence Sentinel Survey, http://www.nigeriaaids.org/documents/2010_National\%20HIV\%20Sero\%20Prevalence\%20Sentinel\%20Surveypdf 2010.

[3]. Egger M, May M, Chene G, Phillips AN, Ledergerber B, Dabis F, et al. Prognosis of HIV-1-infected patients starting highly active antiretroviral therapy: a collaborative analysis of prospective studies. Lancet. 2002;360:119-29. PubMed Abstract | Publisher Full $\underline{\text { Text }}$

[4]. Jahn A, Floyd S, Crampin AC, Mwaungulu F, Mvula H, Munthali F, et al. Population-level effect of HIV on adult mortality and early evidence of reversal after introduction of antiretroviral therapy in Malawi. Lancet. 2008;371:1603-11)

[5]. Calmy A, Klement E, Teck R, Berman D, Pecoul B, FerradiniL. Simplifying and adapting antiretroviral treatment in resource-poor settings: a necessary step to scaling-up. AIDS.2004;18:2353-60.

[6]. Wester CW, Kim S, Bussmann H, Avalos A, Ndwapi N, Peter TF, et al. Initial response to highly active antiretroviral therapy in HIV-1C-infected adults in a public sector treatment program in Botswana. J Acquir Immune Defic Syndr. 2005; 40:336-43.

[7]. Wools-Kaloustian K, Kimaiyo S, Diero L, Siika A, Sidle J, Yiannoutsos CT, et al. Viability and effectiveness of large-scale HIV treatment initiatives in sub-Saharan Africa: experience from western Kenya. AIDS. 2006;20:41-8.

[8]. Rosen S, Fox MP, Gill CJ. Patient retention in antiretroviral therapy programs in sub-Saharan Africa: a systematic review. PLoS Med. 2007;4:e298.

[9]. Yu JK, Chen SC, Wang KY, Chang CS, Makombe SD, Schouten EJ, et al. True outcomes for patients on antiretroviral therapy who are 'lost to follow-up' in Malawi. Bulletin of the World Health Organization. 2007;85:550-554. 
[10]. C. A. Onoka, B. S.Uzochukwu, O. E. Onwujekwe, C. Chukwuka, J. Ilozumba, C. Onyedum, E. A. Nwobi, C. Onwasigwe. Retention and loss to follow-up in antiretroviral treatment programmes in southeast Nigeria. Pathogenes and Global Health. 2012 Mar; 106(1) : 46-54.

[11]. Fox MP, Rosen S. Patient retention in antiretroviral therapy programs up to three years on treatment in sub-Saharan Africa, 20072009: systematic review. Trop Med Int Health. 2010;15(Suppl 1):1-15.

[12]. Hanson K, Hicks C. New antiretroviral drugs. Curr HIV/AID Rep. 2006;3:93-101

[13]. Rosen S, Fox MP, Gill CJ. Patient retention in antiretroviral therapy programs in sub-Saharan Africa: a systematic review. PLoS Med. 2007;4:e298.

[14]. Stringer JS, Zulu I, Levy J, Stringer EM, Mwango A, Chi BH, Mtonga V, Reid S et al . Rapid scale-up of antiretroviral therapy at primary care sites in Zambia: feasibility and early outcomes. . JAMA. 2006;296(7):782-793.

[15]. Kenneth A, Uche O, Azuka O, Obialunamma O. Treatment outcomes in patients receiving combination antiretroviral therapy. Retrovirology. 2010; 7( 1): 54

[16]. Nglazi D M, Kranzer K, Holele P,Kaplan, Mark D, Jaspan H et al, Treatment outcomes in HIV-infected adolescents attending a community-based antiretroviral therapy clinic in South Africa BMC Infectious Diseases 2012, 12:21

[17]. Bygrave H, Mtangirwa J, Ncube K, Ford N, Kranzer K, Munyaradzi D. Antiretroviral Therapy Outcomes among Adolescents and Youth in Rural Zimbabwe. PLoS One. 2012; 7(12)

[18]. S.I. Bello Management Outcomes of HIV/aids Patients on Haart in a Secondary Health Institution in North Central, Nigeria Pharmacologia 2012 3(8): 336-343

[19]. Agu, K.A., U.M. Ochei, A.C. Oparah and O.U. Onoh, 2010. Treatment outcomes in patients receiving combination ARV therapy in Central hospital, Benin City, Nigeria. Trop. J. Pharm. Res., 9: 1-10

[20]. Amusa, Y.B., A.O. Adisa, I.A. Adediran and M.A. Dunsimi, 2004. Otorhinolaryngologic associated features of HIV/AIDS patients in Ile-Ife, Nigeria. Niger. J. Clin. Pract., 7: 69-73

[21]. . Boulle A, Van Cutsem G; Hilderbrand K; Cragg C; Abrahams, M; Mathee, S; Ford et al . Seven-year experience of a primary care antiretroviral treatment programme in Khayeli sha, South Africa. AIDS, 2010;24(4) :563-572.

[22]. . Schöni-Affolter F, Keiser O, Mwango A, Stringer J, Ledergerber B, Mulenga L et al. Estimating Loss to Follow-Up in HIVInfected Patients on Antiretroviral Therapy: The Effect of the Competing Risk of Death in Zambia and Switzerland. PLoS One. 2011; 6(12): e27919

[23]. Jeffrey S. A. Stringer, Isaac Zulu,Jens Levy, Elizabeth M. Stringer, Albert Mwango, Benjamin H. Chi, Vilepe Mtonga, et al. Rapid Scale-up of Antiretroviral Therapy at Primary Care Sites in Zambia: Feasibility and Early Outcomes. JAMA. 2006 Aug 16;296(7):782-93 